\title{
Stratégie industrielle et développement régional : le cas de la Mauricie au $\mathrm{XX}^{\mathrm{e}}$ siècle
}

\section{Pierre Lanthier}

Volume 37, numéro 1, juin 1983

URI : https://id.erudit.org/iderudit/304122ar

DOI : https://doi.org/10.7202/304122ar

Aller au sommaire du numéro

\section{Éditeur(s)}

Institut d'histoire de l'Amérique française

\section{ISSN}

0035-2357 (imprimé)

1492-1383 (numérique)

Découvrir la revue

\section{Citer cet article}

Lanthier, P. (1983). Stratégie industrielle et développement régional : le cas de la Mauricie au XX ${ }^{\mathrm{e}}$ siècle. Revue d'histoire de l'Amérique française, 37(1), 3-19. https://doi.org/10.7202/304122ar d'utilisation que vous pouvez consulter en ligne. 


\title{
STRATÉGIE INDUSTRIELLE ET DÉVELOPPEMENT RÉGIONAL: LE CAS DE LA MAURICIE AU XX ${ }^{\mathrm{e}}$ SIËCLE ${ }^{1}$
}

\author{
PIERRE LANTHIER* \\ Groupe de Recherches sur la Mauricie \\ Université du Québec à Trois-Rivières
}

\begin{abstract}
À la veille des années trente, la Mauricie était devenue une région industrielle bien en vue non seulement au Québec, mais en Amérique du Nord. Son potentiel hydro-électrique et ses usines de pâtes et de papier journal se rangeaient parmi les plus importants dans le monde, sans compter que ses entreprises de textile, de vêtement, de chimie et d'électro-métallurgie employaient plusieurs milliers de personnes.
\end{abstract}

Historiquement et géographiquement, l'industrialisation de la Mauricie s'est accomplie tout d'abord le long de la rivière Saint-Maurice: à partir de 1882, sur le territoire de Grand'Mère et de Shawinigan; après 1906, à Trois-Rivières et au Cap-de-la-Madeleine; entre 1907 et 1910, dans la municipalité de La Tuque. Puis, dans l'entre-deux-guerres,

\footnotetext{
* Cet article s'inscrit dans le cadre des activités menées par le Groupe de Recherches sur la Mauricie (Université du Québec à Trois-Rivières). L'auteur tient à remercier $\mathrm{M}$. Normand Séguin pour les conseils et avis fournis pendant la recherche et la rédaction, et les organismes financiers suivants: FCAC, le Conseil de recherches en sciences humaines du Canada, le FIR de l'Université du Québec à Trois-Rivières, la Société St-Jean-Baptiste de la Mauricie ainsi que M. René Hardy pour ses démarches auprès de ces organismes.

Ce travail repose sur un dossier que l'auteur et M. Alain Gamelin ont constitué en 1980 81: L'industrialisation de la Mauricie, dossier statistique et chronologique, 1870-1975 (TroisRivières, 1981). Publication du Groupe de recherche sur la Mauricie (UQTR), cahier no 6, 490 p. Ce dossier a été élaboré à partir de fort nombreuses sources qu'il n'est pas possible de citer entièrement ici. On consultera le cahier pour en avoir la liste. Mentionnons toutefois les plus importantes: ainsi les différentes publications gouvernementales, comme les Recensements $d u$ Canada. les Annuaires du Québec, Annuaires du Canada, les dossiers du Bureau Fédéral de la Statistique: les rapports annuels des principales entreprises établies en Mauricie, l'Annual Financial Review, le Survey of Corporate Securities du Financial Post, le National Directory of the Pulp and Paper Industrv of Canada, le Manual of the Textile Industries of Canada; bien entendu, des ouvrages comme ceux de B. Brouillette, Le développement industriel de la Vallée du SaintMaurice (1932), R. Blanchard, La Mauricie (1950), J.H. Dales, Hydroelectricity and Industrial Developpement: Quebec, $1898-1940$ (1957), W. Ryan, The Clergy and Economic Growth in Quebec (1896-1914) (1960) ont été utilisés, de même que les thèses suivantes: N. Brouillette, Le déclin industriel de Shawinigan (1972). J. Belleau, L'industrialisation de Trois-Rivières (19051925) (1979), A. Dion, L'industrie des pâtes et papiers en Mauricie (1887-1929) (1981), F. Uren, The Historical Geography of the St. Maurice Valley with Special Reference to Urban Occupancy (1949). R. Parent, Wayagamack Pulp and Paper Co. Ltd (1910-1929) (1974). Il ne faut pas oublier les ouvrages d'érudits locaux comme F. Larochelle, Shawinigan depuis 75 ans (1976). Le Nouvelliste de Trois-Rivières s'est révélé une source utile, de même que la documentation fournie par les compagnies.
}

RHAF, vol. 37, no 1, juin 1983 
Louiseville et, dans une moindre mesure, Saint-Tite ont joint les rangs des villes industrielles.

La crise de 1929 a freiné la croissance industrielle de la région. En revanche, la deuxième guerre mondiale lui a donné vigueur au point que tout semblait alors à nouveau prometteur. Cependant, à partir des années cinquante, l'expansion s'est essoufflée. Toutes les villes, sauf Trois-Rivières, ont vu leur nombre d'employés dans le secteur manufacturier plafonner à cette époque. Trois-Rivières n'y échappait plus après 1965. Pour sa part, la ville de Shawinigan subissait même un net déclin.

Que s'est-il passé? Diverses explications ont été avancées, parmi lesquelles les plus fréquemment citées pointent du doigt la croissance du mouvement syndical dans les années quarante et cinquante et l'étatisation de l'électricité en 1963. Il n'est pas question de tester ici la solidité de ces arguments, ni même d'apporter une réponse définitive à la question. Il s'agit plutôt de mettre de l'avant un élément qui a été jusqu'ici négligé dans le débat: les stratégies industrielles adoptées par les grandes entreprises.

On rassemble généralement ces stratégies en trois grands types: l'intégration horizontale, l'intégration verticale et la diversification. En quoi consistent-ils?

1. L'intégration horizontale se rencontre lorsqu'une entreprise veut accroître sa capacité de production dans un secteur bien déterminé soit en créant des usines, soit en absorbant celles de ses concurrents. Par exemple, lorsqu'une société de papier journal construit de nouvelles unités de production ou achète ses concurrents tout en se maintenant dans la fabrication du papier journal.

2. L'intégration verticale apparaît lorsqu'une entreprise décide de concurrencer ou de racheter ses fournisseurs et/ou ses clients. Ainsi, quand une société, en plus du papier journal, se met à fabriquer de la pâte à papier ou à imprimer des journaux.

3. Enfin, on dit qu'il y a diversification lorsqu'une société, désireuse de déborder son secteur de production, se lance dans un ou plusieurs secteurs parallèles. Une entreprise de papier journal peut diversifier sa production en y ajoutant la cartonnerie, l'emballage, le papier hygiénique, etc. À l'intérieur de ce processus, elle peut même aller audelà de la pâte et du papier et se lancer, par exemple, dans l'industrie du bois ou du verre. Cette entreprise devient alors un conglomérat.

L'adoption de l'une ou de l'autre de ces stratégies est rarement le fait d'une seule entreprise. Elle résulte des conditions et des possibilités du financement et du marché. C'est pourquoi tel type de stratégie peut être choisi non seulement par les sociétés d'un même secteur industriel, 
mais aussi par les entreprises n'ayant entre elles aucune parenté de production. Ce phénomène n'est pas sans entraîner certaines conséquences sur le développement régional.

Il est remarquable de constater, en effet, que les phases de croissance et de plafonnement sont accompagnées et, le plus souvent, précédées de décisions et d'investissements modifiant l'orientation structurelle des groupes implantés en Mauricie. C'est ce dont il sera question dans le présent article. Dans une première partie, le rôle primordial de la Shawinigan Water \& Power (SWP) dans le développement régional sera mis en relief; après quoi l'on tiendra compte des stratégies adoptées par les grandes sociétés.

\section{Le rôle moteur de la Shawinigan Water and Power}

Entre 1882 et 1887, John Forman a lancé la Laurentide Pulp Co. à Grand'Mère. Cette entreprise fut la première dans la région à exiger à la fois une main-d'oeuvre abondante et une technologie avancée. Toutefois, cette implantation ne pouvait seule assurer le démarrage régional.

Le développement de la Mauricie a avant tout reposé sur le dynamisme d'une compagnie de production et de distribution d'électricité, la Shawinigan Water \& Power (SWP). Cette société, d'origine américaine, n'a pas hésité à s'endetter lourdement à moyen et à long terme afin de financer des immobilisations particulièrement importantes pour l'époque. La logique de sa stratégie était simple: l'exploitation d'une centrale électrique n'est rentable que si elle est de grande puissance. Or les chutes de Shawinigan offraient la possibilité de construire pareille centrale.

Il restait à trouver un marché à la mesure du projet. À cette époque, deux possibilités se présentaient: la ville de Montréal et la grande industrie. Pour rejoindre la première, il fallait des investissements aussi considérables que ceux requis par la construction de la centrale et surtout beaucoup de temps. Quant à la seconde, la Mauricie était une région trop neuve pour posséder un nombre appréciable d'entreprises à grande consommation d'énergie.

C'est pourquoi les dirigeants de la SWP, tout en construisant la première centrale, partirent à la recherche d'entreprises susceptibles d'ériger dans la région de Shawinigan des usines exigeant une consommation considérable d'énergie. Et, en 1898, T. Willson prenait la décision, sous l'influence de la SWP, de construire une usine de carbures à Shawinigan. En 1900, il signait avec la compagnie d'électricité un contrat pour la fourniture de $5000 \mathrm{HP}$. À son tour, en 1899, la Pittsburg Reduction (aluminium), n'ayant pu obtenir la fourniture importante d'énergie des chutes du Niagara, se laissait convaincre par la SWP de 
venir installer son aluminerie à Shawinigan. Entre les deux compagnies fut alors établi un contrat pour la fourniture de $5000 \mathrm{HP}$ en énergie hydraulique. En 1900, un autre contrat impliquant de l'énergie hydraulique ratifia une entente entre la SWP et des hommes d'affaires belges voulant construire une usine de pâtes à Shawinigan. Comme on peut le voir, les efforts déployés par la SWP ont porté fruit. Mais pas de manière satisfaisante: sur les trois contrats, deux impliquaient de l'énergie hydraulique et n'apportaient donc pas de clients à la centrale hydroélectrique de Shawinigan. Le problème de la vente des excédents se posait donc toujours avec acuité; et d'autant plus que l'usine de la Shawinigan Carbide, avant 1914, était trop petite pour constituer un marché intéressant. Lorsqu'on examine la production d'énergie de la SWP, on constate que l'énergie hydraulique a occupé une place aussi importante (sinon plus) que l'énergie électrique avant la Première Guerre Mondiale:

\section{Énergie hydraulique}

1904

1907

1909

1912

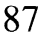

168

199

257
Énergie électrique

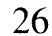

60

151

277

(en millions de $\mathrm{KW} / \mathrm{h}$ )

En fait, on peut supposer que l'arrivée de ces sociétés à Shawinigan aura permis à la SWP d'obtenir tout juste de quoi assurer son fonds de roulement. Comme l'a indiqué J.H. Dales ${ }^{2}$, ce n'est que lorsque les lignes de la SWP atteignirent Montréal en 1903 que l'entreprise devint solvable et put songer à s'étendre davantage.

Le marché montréalais devenait donc le client le plus sérieux de la compagnie. En fut-il de même par la suite? Certes, jusque dans les années 1940, la consommation régionale d'électricité pour usage domestique, municipal et rural ne comptait guère que pour $2 \%$ des ventes totales (en $\mathrm{KW} / \mathrm{h}$ ) de la compagnie. Mais la vente aux sociétés de distribution (dont celles de Montréal) a régressé de 30,7\% en 1925 à $21,4 \%$ en 1940 , tandis que les ventes totales passaient de 1,53 à 3,73 milliards de $\mathrm{KW} / \mathrm{h}$. Dans l'entre-deux-guerres, l'électro-chimie et l'électro-métallurgie (avec $21,2 \%$ des ventes en 1940), et surtout la pâte et le papier $(48,3 \%)$ avaient pris la part la plus considérable ${ }^{3}$. Il s'agissait là d'entreprises régionales.

\footnotetext{
2 J.H. Dales, Hydroelectricity and Industrial Development: Quebec, 1898-1940 (Cambridge, 1957), 54 .

J.H. Dales, op. cit., 84 ss. En 1920 la charge consommée par la Montreal Light, Heat and Power s'élevait à $29,3 \%$ de la charge totale, et les autres compagnies de distribution s'accaparaient de $6,5 \%$; soit en tout $35,8 \%$. (Source: Rapport annuel de la SWP pour 1920).
} 
La SWP avait continué de tout mettre en oeuvre pour attirer divers établissements industriels dans l'orbite de ses installations. Ainsi, en 1921, ses ingénieurs mettaient au point une chaudière électrique de grande capacité devant remplacer les chaudières à charbon dans les entreprises à forte consommation énergétique. Cette chaudière venait à point: la guerre de 1914-18 a en effet accru dans des proportions gigantesques le prix mondial du charbon. La chaudière électrique fut sans doute un argument majeur pour amener des entreprises de pâtes et papiers comme l'International Paper et la St. Lawrence Paper Mills Ltd à venir s'établir à Trois-Rivières dans les années vingt. Dans le domaine des abrasifs, l'électricité a attiré la Canadian Carborundum à Shawinigan en 1917.

En fait, comme l'atteste ce commentaire tiré du rapport annuel de 1932, la SWP avait développé une véritable stratégie de développement industriel:

Depuis plusieurs années, la compagnie a maintenu un département dans le but de découvrir des industries qui pourraient profitablement être développées dans le territoire de la compagnie. Durant l'année écoulée, ce département a été actif, et a obtenu un certain succès. Aucune industrie importante n'a été fondée mais bon nombre de petites compagnies se sont établies dans les limites de la zone d'influence de la compagnie.

Il est certain que le dynamisme manifesté par la SWP explique pour une bonne part l'expansion de la région. Il conviendrait toutefois de ne pas en faire le facteur unique. Déjà, avant même que la grande industrie ne vienne s'y implanter, de nombreux établissements d'importance moyenne faisaient vivre la région. Par exemple, dans le domaine du cuir, on peut mentionner la ganterie Balcer (1854) et la Tebbutt Shoes (1888); dans celui du bois, les établissements Girard et Godin (fabrication de cercueils); et dans les métaux la Compagnie canadienne des Conduits (1889), qui devint la Montreal Pipe \& Foundry en 1903 puis la Canadian Iron Foundry en 1908. Ces établissements employaient un minimum de cent employés chacun.

D'autres groupes industriels ont exercé une influence régionale notable. À Trois-Rivières, le groupe Whitehead (Wabasso en 1907 et Wayagamack en 1911) a servi de moteur initial au développement de la grande entreprise trifluvienne. À La Tuque, ce fut la Brown Corporation (1907).

Enfin et surtout, indépendamment des influences individuelles et directes, la plupart des grandes entreprises venues s'implanter dans la Mauricie ont suivi un ensemble de stratégies, avant 1940, qui a encouragé la venue de sociétés nouvelles. 


\section{Les stratégies des grands groupes industriels}

En injectant dans la Mauricie d'énormes capitaux, les groupes industriels n'ont pas seulement contribué à développer la région, mais aussi à la rendre plus dépendante d'autres régions. Plus la Mauricie se développait, et plus elle dépendait des marchés national et international. Or cette dépendance était fonction des intentions des groupes industriels quant à leur manière de conquérir ces marchés. Dans l'ensemble, on s'aperçoit que ces stratégies ont suivi deux phases: 1- de 1910 à 1940, une période d'intégration verticale limitée accompagnée d'une décennie d'intégration horizontale; 2- de 1940 à 1971, intégration horizontale et diversification.

\section{a) La période 1910-1940}

À première vue, il serait aisé de concevoir que l'industrie mauricienne, durant ses premières années, s'est contentée de transformer la matière première en des produits semi-finis qu'elle exportait en dehors de la région. La situation n'était toutefois par aussi simple. Suivant les possibilités de la main-d'oeuvre et du marché, les entreprises de la Mauricie ont tenté de gravir quelques échelons dans la fabrication du produit semi-fini afin de dépasser le strict stade de la transformation de la matière première. Ce fut surtout le cas avant 1925. Par la suite, et quelquefois en même temps, elles ont essayé d'accroître leurs moyens de production. Examinons ces deux mouvements à l'aide d'exemples.

Tout d'abord, l'intégration verticale. Un premier exemple est celui de la SWP. Tout en développant ses centrales de production d'énergie électrique et en acquérant le contrôle graduel de petites compagnies de distribution d'électricité, donc tout en poursuivant une concentration horizontale, le groupe SWP a été amené à s'intéresser à l'industrie des carbures par l'acquisition d'une filiale en 1908-09, la Shawinigan Carbide. Il s'agissait là de l'absorption d'un client important. À noter toutefois que l'absorption ne fut pas complète, en ce sens que la filiale chimique n'a jamais été réduite à une division de la SWP et conserva toujours son statut juridique de société. En outre l'organisation interne et le personnel dirigeant de la filiale chimique bénéficièrent toujours d'une large autonomie à l'intérieur du groupe ${ }^{4}$. En fait, la mise en filiale de la Shawinigan Carbide avait des motifs d'ordre financier: cette compagnie s'était installée à Shawinigan dans le but de produire du gaz acétylène destiné à l'éclairage. Or l'électricité ayant mis fin à ces espoirs, une reconversion était d'autant plus difficile à accomplir que la concurrence américaine dans ce secteur se faisait lourdement sentir. D'où l'absorption par la SWP qui lui assura des garanties financières et l'in-

\footnotetext{
4 Sur cette question, voir A.F.G. Cadenhead, History of Shawinigan Chemicals Ltd, 19l61946 (Shawinigan, 1946), manuscrit fourni par la Gulf Canada Co. au Groupe de recherche sur la Mauricie.
} 
cita dès 1908 à se lancer dans la recherche de nouveaux débouchés pour sa production. Et dès 1912, un nouveau procédé de soudure et de coupe du métal était mis au point, le prestone.

C'est toutefois pendant la première guerre mondiale que la Canada Carbide prit une expansion considérable en trouvant de nouveaux produits (dont l'acétone) et en lançant le magnésium métallique, le ferrosilicium et même la production d'électrodes. Elle créa à cette fin trois filiales qu'elle absorba en 1927 pour devenir la Shawinigan Chemicals. Et en 1928, elle lança une nouvelle société, la Shawinigan Stainless Steel and Alloys Ltd (duriron et acier inoxydable) qu'elle intégra à ses propres structures l'année suivante. C'est là le cas le plus important d'intégration verticale qu'a connu l'industrie mauricienne des débuts jusqu'à nos jours. On notera toutefois que le processus ne va pas jusqu'au produit fini: il ne fait que gravir un échelon de plus vers le produit fini, sans l'atteindre.

Un autre exemple: le secteur des pâtes et papiers. Tout d'abord, la Laurentide: jusqu'en 1895, cette compagnie ne s'occupa que de pâtes. Cette année-là, il fut décidé d'amorcer une intégration verticale en ajoutant la production de papier journal ${ }^{5}$. Même phénomène à la Belgo en 1900-02: au tout début, la compagnie ne fabriquait que de la pâte; et en 1902, son directeur, H. Biermans, proposa d'ajouter le papier journal. La proposition fut repoussée par les actionnaires, mais Biermans persista dans son projet en créant une société fermière. Et en 1906, la décision fut entérinée par les actionnaires. La St. Maurice Paper, au Cap-de-la-Madeleine, a commencé elle aussi ses activités avec de la pâte seulement (1910) pour ensuite intégrer la fabrication du papier journal en 1916. La Wayagamack, dès sa création (1910-13), se lança dans la pâte et le papier kraft; en 1926, par l'intermédiaire d'une filiale, la Wayagamack News, elle produisit également du papier journal. Pour leur part, les usines trifluviennes de la CIP et de la St. Lawrence Paper Mills, créées respectivement en 1920 et 1923, ont commencé leurs activités à la fois dans la pâte et le papier journal. La seule usine à n'avoir pas été touchée par l'intégration verticale fut celle de la Brown Corporation à La Tuque, qui en resta à la production de la pâte. Toutefois elle améliora la technologie de la fabrication de la pâte et accrut ses établissements en conséquence. Lorsque les frères Brown construisirent l'usine en 1907, ils avaient l'intention de l'intégrer à celle qu'ils possédaient déjà à Berlin (New-Hampshire): l'usine de La Tuque fabriquerait de la pâte, et celle de Berlin la transformerait en papier. Donc un processus d'intégration verticale à l'intérieur du groupe. C'est ce processus qui a fait vivre la ville pendant une cinquantaine d'années. Le secteur des pâtes et papiers fut donc lui aussi témoin d'une intégra-

\footnotetext{
5 Sur ce point, voir J. Niosi, «La Laurentide (1887-1928): pionnière du papier journal au Canada", in Revue d' histoire de l'Amérique française, 29, 3 (déc. 1975): 375-415.
} 
tion verticale, mais bornée surtout au papier journal. Et encore cette intégration n'alla pas jusqu'au contrôle des principaux clients du papier journal.

En général, l'intégration verticale fut limitée et devait permettre aux sociétés d'accroître horizontalement leurs activités au niveau de ce qu'elles avaient intégré verticalement. Il n'était pas question de poursuivre l'intégration jusqu'au produit final, mais plutôt de contrôler le marché des produits intermédiaires. Somme toute, l'intégration verticale ne faisait que précéder (ou accompagner) une poussée horizontale plus importante.

Une exception toutefois: la Wabasso Cotton Ltd. Ayant ouvert à Trois-Rivières une usine pour cotonnades fines et blanches en 1907, puis une filature à Shawinigan en 1910, le groupe créa, en 1910 toujours, trois petites filiales de fabrication de vêtements: la Diamond Whitehead à Trois-Rivières (sous-vêtements, vestes et chemises), la Shawinigan Knittings à Shawinigan (produits en tissu maillé) et la Oxford Knittings à Woodstock en Ontario (bonneterie et tricots). L'existence de ces trois filiales fut éphémère: la Diamond Whitewear ferma ses portes en 1920, la Shawinigan Knittings dut louer ses locaux à J.R. Moodie dès avant 1914, et la Oxford Knittings disparut durant les années vingt. Cette intégration n'a donc pas fonctionné. Elle avait été poussée trop loin dans le sens du produit fini. L'économie régionale ne semblait pas pouvoir absorber un tel processus. Plus tard cependant, la Wabasso renouvela sa tentative, en se limitant cette fois-ci au niveau intermédiaire: entre 1924 et 1927 , elle introduisit à Trois-Rivières une usine d'impression et de teinturerie et utilisa les locaux de l'ancienne Diamond Whitewear pour la fabrication de la soie artificielle et le mercerisage.

Mais parallèlement à l'intégration verticale, la Wabasso en a profité pour doubler sa capacité de production à Trois-Rivières en 191213, effectuer d'importants agrandissements de sa blanchisserie et de son usine à tisser en 1923-24, et accroître sa productivité en remplaçant en 1929 son vieil équipement (d'origine britannique) par de la machinerie automatique américaine. Il s'agissait donc d'une expansion horizontale. Or cette stratégie fut plus importante sur le plan financier et des équipements que les essais d'intégration verticale.

C'est d'ailleurs cette concentration horizontale qui devint le fait majeur des années vingt en Mauricie. La guerre de 1914-18 a provoqué une expansion sans précédent de l'industrialisation. Mais elle a également, de par le caractère exceptionnel et temporaire de la demande, créé une concurrence impitoyable sur le plan international. Vu la faiblesse des marchés canadiens, cette concurrence a mis fin à l'intégration verticale des groupes et amené les sociétés à des ententes permettant de nouvelles implantations dans la chimie, et à la concentration 
horizontale dans les pâtes et papiers, l'aluminium, l'électricité, etc. Les pâtes et papiers virent, dans les années vingt, augmenter de manière disproportionnée (par rapport au marché) la capacité de production: ceci poussa les compagnies de Grand'Mère, Shawinigan, du Cap-de-laMadeleine, la Wayagamack (Trois-Rivières) et Port-Alfred à abandonner leurs entités respectives et à former, de 1926 à 1931, la Consolidated Paper ${ }^{6}$. Pour sa part, la CIP accrut considérablement ses moyens de production à Trois-Rivières et en profita pour absorber la Riordon Co. Ltd (propriétaire d'usines à Kipawa, Gatineau, Riordon, Hawkesbury et Calumet).

De son côté, la Northern Aluminium (Alcan), après avoir construit deux usines de transformation (ustensiles de cuisine et laminage de tôle d'aluminium) à Toronto en 1913 et en 1915, érigea dans les anriées vingt à Arvida la plus grosse aluminerie du monde. La SWP, en plus de construire ses propres centrales hydro-électriques, en profita pour prendre le contrôle technique et financier des centrales des compagnies de pâtes et papiers de Grand'Mère et de La Tuque. La Canada Iron Foundries suivit un processus semblable: après s'être débarassée de ses hauts fourneaux, elle se concentra horizontalement dans la fonderie; à cette fin, elle créa (ou racheta) des fonderies en Ontario et s'orienta dans la tuyauterie et la machinerie. Enfin, à Shawinigan, les filiales de la Canada Carbide ont eu la vie dure après la guerre, par suite de la concurrence mondiale. La compagnie les absorba en 1927, afin d'assouplir ses structures et accentuer la recherche et la mise en marché rapide des nouveaux produits.

Somme toute, pour répondre à une conjoncture fortement concurrentielle, on augmenta la capacité de la production (plus grande est la taille et plus économique est le prix de revient), ou encore on la concentra dans des unités plus grandes ${ }^{7}$. Et pour compléter le processus, on préféra inviter fournisseurs et clients en Mauricie plutôt que de les concurrencer. Enfin, on créa des filiales exclusivement versées dans la vente des produits, ou, à tout le moins, des agences de vente. En 191920, la Canada Carbide créa la Shawinigan Ltd à Londres (G.-B.) et la Shawinigan Products Corp. à New York en vue de pénétrer les marchés anglais et américain. En 1916, la Laurentide et la Belgo participèrent à la création de la Canadian Export Paper Co. La St. Maurice Paper signa un accord avec cette société en 1917. En 1926, la St. Lawrence créa la St. Lawrence Sales Co. Ltd; en 1927, la Canada Iron lança une filiale de vente, la Paper Machinery Ltd.

\footnotetext{
6 Voir G. Piédalue, «Les groupes financiers et la guerre du papier au Canada, 1920-1930», in Revue d' histoire de l'Amérique française, 30, 2 (sept. 1976): 223-258.

7 Ce phénomène n'est en aucune manière propre à la Mauricie, ni même au continent nordaméricain. L'industrie électrique française a connu une situation semblable, et pour les mêmes raisons, dans les années vingt. Voir sur ce point $\mathrm{P}$. Lanthier, Le rôle des groupes industriels internationaux dans l'électrification de la France, 1880-1940, thèse en cours de rédaction et devant être présentée à l'Université de Paris-X (Nanterre).
} 
Les conséquences de ces stratégies sur la croissance de la région furent décisives. L'intégration verticale limitée des groupes s'est accomplie exclusivement en Mauricie. Pour sa part, l'expansion horizontale a débordé les limites régionales. Cependant, elle a quand même attiré de nombreux clients et fournisseurs sur le territoire mauricien. Ceci de trois façons:

1- Les différents processus d'intégration ont suscité dans la région des investissements d'une telle importance qu'il devenait rentable pour certains fournisseurs de s'y établir ou d'y demeurer. Deux exemples: la Canada Iron Foundries et la George Christie. La première possédait déjà une usine à Trois-Rivières. Celle-ci a brûlé dans l'incendie qui ravagea la ville en 1908. Ses dirigeants eurent alors l'intention de reconstruire à Farnham. Mais l'intervention de Whitehead, une exemption de taxes et des tarifs préférentiels d'électricité de la part de la SWP ont convaincu le groupe de reconstruire à Trois-Rivières. Bien lui fit, car cette société devint l'un des principaux fournisseurs en machineries et tuyauteries diverses des entreprises régionales. De son côté, la George Christie (devenue la Niagara Wires en 1937) s'implanta à Trois-Rivières en 1923. Sa spécialité: fabriquer des toiles métalliques pour les entreprises de pâtes et papiers.

2- Les agrandissements et les implantations d'usines amenèrent dans les villes de la Mauricie une main-d'oeuvre masculine importante et, avec elle, son complément féminin pouvant être employé dans des entreprises utilisant une main-d'oeuvre à bon marché. Notamment, dans les années vingt, l'arrivée et l'accroissement à Trois-Rivières de sociétés de pâtes et papiers permirent à de nombreuses compagnies spécialisées dans le vêtement de s'installer dans cette ville ou au Cap-de-laMadeleine. C'est à cette occasion que la Fergusson Atlantic ouvrit ses portes à Trois-Rivières. Phénomène semblable à Shawinigan et à Grand'Mère: les établissements de chimie et de pâtes et papiers rendirent possible la venue à Grand'Mère de petites entreprises spécialisées dans le vêtement et le cuir. Dans une proportion très importante, les PME régionales de cette catégorie étaient francophones et d'origine locale ${ }^{8}$. Ce fut le cas de la Grand'Mère Knittings, lancée par A. Thibault en 1933 et des nombreuses entreprises de cuir à Saint-Tite.

3- Très souvent, plutôt que de poursuivre une intégration verticale dans des secteurs nouveaux ou exigeant des investissements importants, les entreprises de la région ont préféré inviter certains de leurs clients à venir en Mauricie.

Dans le secteur chimique, tout en se dotant de laboratoires de recherche importants, la Canada Carbide (qui a succédé à la Shawinigan

8 Il n'a pas été possible, à ce stade-ci de la recherche, de mesurer avec exactitude l'importance de ces PME. C'est plutôt la simple observation de multiples cas qui permet d'avancer cette constatation. 
Carbide en 1911 et qui allait devenir la Shawinigan Chemicals en 1927) chercha à rentabiliser les produits nouveaux mis sur le marché. À cette fin, elle attira à Shawinigan deux sociétés: la Prest-O-Lite (1917) et la CIL (1931). Et sans doute ne fut-elle pas étrangère, grâce à ses recherches du côté de la rayonne (1919-29), à l'implantation de l'Associated Textile à Louiseville en 1929 et de la Laurentian Silk Mills au Cap-de-laMadeleine en 1928; et même au développement d'une division de mercerisage et de soie artificielle à la Wabasso dans les années vingt.

Dans les pâtes et papiers, l'exemple de la Bates Valve Bags mérite d'être mentionné: implantée au Cap-de-la-Madeleine en 1926 et devenue la St. Regis Paper en 1930, cette société achetait du papier kraft de la Wayagamack pour fabriquer des sacs.

Enfin, tout ce processus a été mené par un patronat qui ne s'est pas contenté d'accroître la rentabilité de ses usines, mais qui s'est également intéressé au développement régional en tant que tel. Des hommes comme E. Aldred (SWP) et C. Whitehead (Wabasso) se sont particuliètrement illustrés dans ce domaine. Ainsi, le premier s'est occupé de cités-jardins à Shawinigan, sans compter qu'il a permis au Québec de créer sa première école technique ${ }^{9}$.

Ceci dit, peut-on attribuer à la concentration horizontale les déboires économiques des années trente? On a beaucoup insisté sur les aspects financiers de la crise de 1929 (krash boursier, difficultés monétaires, endettement des pays, etc.). Et pourtant, la crise n'était pas que financière. Le seul fait de sa durée devrait suffire à le démontrer. La guerre de 1914-18, en redistribuant les cartes financières internationales, a également suscité dans l'ensemble des pays une concurrence sans précédent, comme on l'a vu. Et le processus d'expansion et de concentration horizontales n'a-t-il pas provoqué un état de surinvestissement, notamment dans le secteur des produits semi-finis? Et cette congestion n'a-t-elle pas entraîné à son tour la paralysie de l'économie, du fait que les capitaux investis ne pouvaient plus être convenablement rémunérés? C'est là une hypothèse à vérifier.

Quoi qu'il en soit, une chose est certaine: la crise des années trente allait remettre en question toute la stratégie industrielle mauricienne.

\section{b) De 1940 à 1975}

La crise qui a traversé le monde entre 1929 et 1939 a porté un coup particulièrement dur aux entreprises qui ont cru consolider leur position en pratiquant une concentration horizontale. À court terme, cette concentration supposait d'énormes dépenses de rationalisation et d'organisation interne des usines absorbées. Elle avait pour but principal de

\footnotetext{
9 C. Bellavance mène présentement une recherche sur le patronat mauricien de 1900 à 1950.
} 
réduire la surproduction et les prix de revient. Or le résultat le plus tangible pour de nombreuses entreprises fut le blocage prolongé de leur expansion. C'est le cas, notamment, de la Consolidated Paper, qui ne reprit ses investissements qu'après 1945; de la Wabasso, qui en fit autant; de la SWP, qui dut ralentir ses projets de construction de centrales en amont de La Tuque.

Après la Deuxième guerre mondiale, les groupes industriels ont cessé de s'étendre horizontalement, à quelques exceptions près: 1- La SWP a poursuivi le contrôle d'entreprises de distribution d'énergie et l'accroissement de son potentiel énergétique par la construction de nouvelles centrales, Shawinigan 3 (1949), la Trenche (1951) et Beaumont (1958) et l'absorbtion d'un concurrent en 1956, la Southern Canada. 2La CIP acheta en 1954 l'usine de la Brown Corp. à La Tuque, mais pour l'intégrer aussitôt dans un processus de concentration verticale. 3Enfin, l'Aluminum Ltd construisit une aluminerie à Kitimat en 1951.

Mais dans l'ensemble, si l'on excepte le cas de la SWP, ces concentrations horizontales ne faisaient qu'accompagner (ou précéder) un processus encore plus vaste: l'intégration verticale et la diversification. Jusqu'en 1930, on accrut le potentiel productif de la région. Succès relatif et incomplet pour les compagnies. Après la crise et la guerre, une nouvelle génération de patrons est apparue dans ces entreprises: des dirigeants qui s'intéressaient de moins en moins à la vie régionale et qui portaient une attention exclusive à la vie des groupes industriels. Qu'il s'agisse d'ingénieurs ayant fait carrière dans le groupe, ou de jeunes diplômés d'universités ou d'écoles commerciales, ils allaient mettre de l'avant la rentabilité de leurs groupes, loin au-dessus des considérations régionales. Or la crise a montré l'insuffisance de la concentration horizontale. Plus intéressant pour eux, la prise de contrôle des clients. Certes, on aurait pu construire en Mauricie des usines de transformation. Mais, 1- le premier résultat aurait été de se lancer dans une concurrence d'autant plus pénible qu'il aurait fallu former des ouvriers qualifiés alors que d'autres régions (plus urbanisées) les possédaient; 2- la Mauricie ne constituait pas un important marché de consommation de masse: il aurait alors fallu s'assurer du transport des produits finis vers les grands centres urbains, ce qui aurait coûté infiniment plus cher que le transport des produits semi-finis. C'est pourquoi on a suivi la politique suivante: absorption des clients là où ils existaient, c'est-à-dire en Ontario et dans la région montréalaise, ou construction de nouvelles usines dans ces régions quand c'était profitable.

L'exemple le plus caractéristique de cette stratégie, c'est celui de l'Alcan: après avoir construit le complexe Kitimat-Kemano, le groupe décida, vers 1957, de s'orienter dans la transformation des produits d'aluminium. Et c'est ainsi que de 1959 à 1975, il investit environ 
$845000000 \$$ dans le secteur de la transformation (allant des pièces pour automobiles jusqu'à la construction en usine de maisons préfabriquées, en passant par les étiquettes et les emballages commerciaux en papier d'aluminium). Le résultat: en 1976, l'Alcan possédait 27 usines, 4 filiales et une participation dans le secteur de la transformation; ces entreprises se répartissaient ainsi: Maritimes: 2; Québec: 9 (dont cinq dans la région montréalaise); Ontario: 15; Ouest Canadien: 6 (dont cinq en Colombie britannique). Ajoutons à ce tableau que le groupe en profita également pour s'étendre considérablement à travers le monde.

Les entreprises de pâtes et papiers ont suivi un itinéraire semblable en diversifiant les débouchés de leurs productions: 1- vers les cartons, les boîtes, les sacs industriels et les contenants en carton, dans le cas de la Consolidated Paper (devenue Consolidated Bathurst en 1967); 2dans le carton, le bois de finition (plywood, masonite) et le papier pour usages divers dont Facelle Royale), dans le cas de la CIP. Et ici aussi, l'Ontario et la région montréalaise ont reçu la part du lion. Il est vrai que les deux groupes ont en outre construit ou acquis de nouvelles usines de pâtes et papiers. Par exemple, Portage-du-Fort dans l'Outaouais (Consolidated Bathurst) ou Matane (CIP). Mais ces expansions dans des régions nouvelles (à cause des réserves forestières?) s'inscrivent dans une stratégie d'intégration verticale.

La Canron a également suivi: elle a acquis force filiales dans le matériel électrique, la charpente, la tuyauterie (en béton et en plastique), le matériel industriel, etc. Sans doute ne s'agit-il pas de produits pour la consommation de masse, mais ce sont des produits finis, dont les usines se retrouvent en Ontario et dans la région montréalaise (Lachine, Ville d'Anjou, etc.).

Ajoutons la Wabasso à ces cas: dans les années cinquante, cette compagnie passa des mains de la famille Whitehead à celles du groupe Woods en Ontario (matériel pour camping). Ce changement, important en soi, amena la Wabasso à produire, entre autres, de grosses toiles et des coutils. Déjà, donc, une intégration verticale s'est produite. Mais le phénomène le plus important pour Trois-Rivières fut sans doute la crise que l'industrie du textile a connue à partir des années soixante, avec les importations croissantes de produits venus du Tiers-Monde et des pays du bloc socialiste. Cette crise poussa la Wabasso à se spécialiser dans le denim, la literie et le fil de coton teint, et à créer une division pour serviettes-éponge à Dunville (Ontario), après l'absorption de la Dominion Fabrics. Donc, intégration vers les produits finis.

L'ensemble de ces processus pourrait expliquer pourquoi la Mauricie n'a pas pleinement profité de l'expansion des années 1950-60. Certes, il y a quand même eu renouvellement des activités industrielles. La seconde guerre mondiale n'a-t-elle pas suscité la création de nouvelles usines par les groupes pionniers? L'Alcan a implanté une fon- 
derie d'aluminium à La Tuque et une seconde aluminerie à Shawinigan. La Shawinigan Chemicals a érigé trois usines, une au Cap-de-la-Madeleine, une deuxième à Shawinigan et une troisième près de Saint-Maurice. Par ailleurs, la St. Regis Paper a agrandi ses établissements à trois reprises $(1937,1946$ et 1950), tandis que la CIP installait en 1946 dans la partie inactive des locaux de la St. Maurice Paper une filiale pour la fabrication des sacs, la Continental Paper. Et surtout, n'a-t-on pas vu l'arrivée de la Reynolds et de la Westinghouse? En 1939, l'International Foils (qui devint la Dominion Foils en 1944, la Rolling Foils en 1951, et enfin la Reynolds Aluminum en 1956) vint s'implanter au Cap-dela-Madeleine. Cette compagnie, qui employait un nombre très important de travailleurs masculins, reçut son complément féminin, si l'on peut dire, avec la venue en 1951 de la Canadian Westinghouse (lampes à incandescence) à Trois-Rivières, qui recrute majoritairement des femmes.

Cependant, les usines de La Tuque et de Saint-Maurice n'ont pas survécu, tandis que l'usine de la Shawinigan Chemicals au Cap fut cédée à la Norton Co. (abrasifs) en 1949, et que la Continental Paper a quitté la région dans la seconde moitié des années cinquante.

Après 1951, aucun groupe industriel étranger à la région n'est venu s'y installer massivement et de manière durable. Mais ceci ne signifie pas que ces groupes ont abandonné la Mauricie comme lieu d'implantation. À partir des années soixante, et plus encore pendant la décennie suivante, des groupes internationaux sont venus s'établir dans la région (surtout dans la partie proche du fleuve), mais avec la taille d'une PME. Par exemple, Bastos et Cegelec à Louiseville, et Sangamo Canada (du groupe Schlumberger) à Trois-Rivières. Bien entendu, ce renouveau est maigre comparé à l'époque précédente, mais il est intéressant en soi, en ce sens qu'il se situe, pour plusieurs de ces sociétés, non plus dans un secteur où priment la matière première et/ou une maind'oeuvre à bon marché, mais dans des activités exigeant un certain niveau de compétence technique de la part des employés.

Enfin, les PME basées sur la main-d'oeuvre à bon marché n'ont pas disparu, loin de là. Si beaucoup parmi elles n'ont eu qu'une existence éphémère, il n'en reste pas moins que la région manifeste une capacité pour le moins remarquable de renouvellement de ces entreprises là où elles sont fortement implantées. Grand'Mère et Louiseville, sur ce point, fournissent d'intéressants exemples.

Donc, La Mauricie de la seconde moitié du XX $\mathrm{XX}^{\mathrm{e}}$ siècle, si elle ne bénéficie plus d'une expansion marquée comme à l'époque des années dix et vingt, ne peut quand même pas être considérée comme une région en déclin. D'ailleurs, en intégrant la clientèle ontarienne et montréalaise, les grandes entreprises mauriciennes n'ont pas entraîné la chute de la région. Au contraire, aussi paradoxal que cela puisse paraître, la 
Mauricie a pu conserver ses usines grâce à ce processus. Car l'intégration a assuré pour les uns, une clientèle permanente aux usines mauriciennes; et pour les autres, une plus grande stabilité financière grâce à la diversification de la clientèle des groupes. L'économie de la région devint dès lors complémentaire des grands centres de production. Ce processus fut en fait la «canadianisation» progressive de tous ces groupes.

Cette préservation de l'économie mauricienne éclate avec d'autant plus d'évidence que nous avons justement l'exemple d'un groupe qui n'a pas suivi le mouvement: la Shawinigan Chemicals. Après 1945, cette société n'a pas tellement cherché à intégrer verticalement. Certes, il faut tenir compte de la Canadian Resins (créée en 1941 pour les besoins de la guerre et reconvertie par la suite dans la fabrication de pellicules filmiques et dans diverses applications de la résine synthétique) et, à partir de 1960, de l'élargissement des activités de la McArthur Chemicals (devenue une filiale en 1958) du côté des produits pharmaceutiques, cosmétiques et alimentaires. Mais l'effort le plus important fut porté ailleurs: la compagnie a en effet préféré réduire le coût de revient de sa production en s'associant avec la B.A. Oil Co. pour se lancer dans la pétrochimie, ceci dès 1951; et créer quelques usines chimiques ici et là aux États-Unis (Californie entre autres), donc loin de son centre de production. La chose peut paraître d'autant plus surprenante qu'avec la guerre, grâce à sa position exceptionnelle en matière de recherches et de brevets, la Shawinigan Chemicals se situait parmi les groupes chimiques les mieux placés au monde. Toutefois, intégrer verticalement suppose la disponibilité d'une masse considérable de capitaux (surtout en chimie). Or, le groupe SWP a estimé que le développement hydro-électrique serait plus avantageux, vue que la pétrochimie, tôt ou tard, finirait par remplacer la chimie classique. C'est pourquoi on a privilégié l'hydro-électricité plutôt que la chimie et collaboré avec une compagnie de pétrole pour se lancer dans la pétrochimie dans la région montréalaise.

Toutefois, à Shawinigan, le groupe a tenté, dans les années cinquante, de reconvertir son secteur chimique en y augmentant la gamme de produits, avec la construction d'usines pour la fabrication de cyanure de sodium, de soude caustique et d'acide sulphurique. On espérait que ces nouveaux venus allaient sauver la chimie shawiniganaise. Par ailleurs, l'on comptait sur les profits tirés de l'hydro-électricité pour développer les marchés de la pétrochimie et des nouveaux produits.

L'étatisation de l'hydro-électricité en 1963, on le comprend alors, fut un véritable désastre pour le groupe SWP - Shawinigan Chemicals. Car une fois les centrales entre les mains d'Hydro-Québec, que restaitil au groupe sinon un secteur chimique dont les travaux de reconversion ont exigé un très lourd investissement et dont l'intégration au marché 
de la consommation de masse était à peine entreprise. Il n'est pas surprenant que le groupe ait éclaté comme de la porcelaine. Et ceci explique également le déclin de Shawinigan: la Shawinigan Chemicals n'étaitelle pas au coeur d'un système de complémentarité de la production qui faisait vivre cette ville?

On peut se demander par ailleurs si le groupe SWP n'aurait pas été bien inspiré de suivre l'exemple de l'International Power \& Paper Co. (formée en 1928). Cette société cumulait elle aussi deux activités exigeant d'importants investissements, l'hydro-électricité et les pâtes et papiers. En 1941, elle s'est scindée en deux parties, l'International Paper et l'International Hydro-Electric System, ce qui a permis à chacune des sociétés nouvelles de se développer sans nuire à la croissance de l'autre. Après la guerre, l'International Paper remit toutes ses filiales canadiennes entre les mains d'une seule, la CIP, qui s'occupa alors de les intégrer et d'en diversifier la production.

Quelque vingt ans plus tard, la Gulf Canada a repris la branche chimique de la SWP. Or elle n'avait que faire des usines de Shawinigan (d'autant plus que déjà l'industrie chimique canadienne entrait dans une crise structurelle). Le pétrole était son intérêt majeur, d'où le sacrifice de Shawinigan. Quant à la pétrochimie de Varennes, la crise pétrolière de 1973 lui a porté un coup dur: en 1975, l'usine cessait de fabriquer le chlorure de vinyl.

\section{Conclusion}

À quoi peut-on attribuer les deux phases d'expansion et de plafonnement observées en introduction? Dans une large mesure, aux stratégies des grands groupes industriels. Tant que ces stratégies reposaient sur un marché à base de produits semi-finis, l'économie mauricienne a bénéficié de la croissance de ses moyens de production. Mais aussitôt que le marché s'est orienté du côté des produits finis, les groupes industriels ont dû suivre en diversifiant leur production et en se rapprochant des grands centres de consommation, d'où le ralentissement de la croissance régionale.

L'ensemble de ces mouvements ont largement contribué à façonner la dualité économique de la région: tout en s'assurant de la disponibilité de la matière ligneuse et d'une importante source d'énergie, les premières sociétés ont suscité la venue d'autres établissements désireux, quant à eux, d'utiliser une main-d'oeuvre nombreuse et bon marché.

Cette dualité mérite attention, car elle révèle l'un des paradoxes du développement mauricien: d'un côté, les besoins en ressources naturelles d'une économie continentale ont été à l'origine de l'industrialisation régionale et lui ont fourni la plus grosse part de son potentiel 
productif. Mais, de l'autre, l'appoint donné par les entreprises à base de main-d'oeuvre à bon marché a assuré une plus grande stabilité à long terme. Ainsi, Shawinigan a vécu trop exclusivement sur la complémentarité de sa production, et lorsque le groupe SWP s'est démantelé après 1963, une grande partie de sa structure industrielle s'est écroulée comme un jeu de dominos. Par contre, à Grand'Mère, à Trois-Rivières et au Cap-de-la-Madeleine, la stratégie industrielle a été axée sur la combinaison matière première/main-d'oeuvre, ce qui a permis une plus grande solidité économique. 